\title{
Reconstruction of Acetabular Wall Using Autologous Bone Graft Following Resection of Pelvic Chondrosarcoma: Surgical Technique and Functional Outcome
}

\author{
Rendy Cahya Soetanto, Muhammad Naseh Sajadi Budi Irawan, Darmadji Ismono \\ Department of Orthopaedics and Traumatology, Medical Faculty, Padjajaran University, Dr. Hasan Sadikin General \\ Hospital, Bandung, Indonesia
}

\section{ARTICLE INFO}

Received : 13 February 2019

Reviewed : 19 March 2019

Accepted : 21 May 2019

\section{Keywords:}

acetabular preservation, bone graft, pelvic chondrosarcoma, functional outcome

\author{
*Corresponding author: \\ Rendy Cahya Soetanto \\ Department of Orthopaedics \& \\ Traumatology, Medical Faculty, \\ Padjadjaran University, Hasan Sadikin \\ Hospital, Bandung, Indonesia \\ Jl. Pasteur No. 38, Bandung, 40161 \\ rendy.cahya@yahoo.com
}

\section{A BSTRACT}

Background: Pelvic chondrosarcoma may be difficult to manage due to its proximities with vital structures. The study aimed to explain an alternative surgical technique for acetabular reconstruction.

Case Presentation: We present a case of a 48-year-old female with large chondrosarcoma of the superior and inferior pubic rami with medial acetabular wall involvement. Pelvic type 3 resection was performed. There was a defect at medial acetabulum after resection. The defect was covered by autograft from iliac and fixation using screws. Rotational pelvic stability was maintained using a reconstruction plate. The functional outcome was assessed 6 months after operation using MSTS and the score was 30 , which was painless, full weight bearing, normal gait, and no pain.

\begin{abstract}
Conclusions: Reconstruction of the pelvis after tumor resection requires a careful preoperative patient evaluation and extensive bone and soft tissue resection to achieve negative tumor margins and stable reconstruction of the osseous and soft tissue defects.
\end{abstract}

\section{INTRODUCTION}

Chondrosarcoma is a malignant main bone tumor that occurs most commonly in the pelvis [1]. Chondrosarcomas are amidst the various regular primary tumors of bones and consists of a cartilaginous membrane without osteoid and are considered for almost $20 \%$ of bone sarcomas [2]. The tumor appears predominantly in the middle-aged people and occurs most generally in pelvic and the upper end of the femur. Although the majority of these tumors present a slow growth rate, rarely metastasize, and have a great prognosis after surgery, local recurrence is a daily occurrence [3].

Chondrosarcoma is confined to the periacetabular region. The hip capacity is compromised. Patient autonomy is directly and significantly changed, which can attend to the patient comprising bedridden. The variety of resection is decided according to the area and grade from the chondrosarcoma. Those resections are represented by the model proposed by Enneking and Dunham. Because of function considerations, fit margins are always the main purpose. Inadequately wide margins, based on Musculo Skeletal Tumor Society
(MSTS) criteria, are predictive of disease recurrence and mortality. However, margins that are over extensive can minimize post-operative function [4].

Ten-year survival of chondrosarcoma grade 2 in the pelvis has been reported by approximately $75 \%$. Recurrence has been reported in $44 \%$ of cases, with a median time to recurrence of 23 months [5]. Pelvic chondrosarcoma is a difficult problem for the orthopedic oncologist. Chondrosarcoma is almost resistant to radiation and chemotherapy. Therefore, a surgical procedure remains the pillar of treatment. Although curettage with local adjuvants is generally treated well for low-grade chondrosarcoma of long bones, most authors advise resection including clear border for pelvic chondrosarcoma of any grades [6,7]. The location of chondrosarcoma in the pelvic has specificities and certain levels of difficulties in surgery because of its location close to vital structure. The depth in pelvis, tumor size, and its relationships with local articular, nervous, vascular and visceral structures usually gain difficult surgical resection and need complex reconstruction [8].

Tumor location is also a determining factor for prognosis. The periacetabular location is statistically a 
low-risk factor for survival. This is due to operator difficulty in obtaining healthy boundaries in certain locations, unlike zone 1 which is accessible, with the exception of crossing sacroiliac articulation [2]. Some surgical options that can be performed on Chondrosarcoma are intralesional surgery, segmental resection, wide resection margins, radical resection, interilioabdominal amputation, reconstruction, and preservation surgery [9].

In 1978, Enneking and Dunham proposed a classification system for pelvic tumor resections: type 1 , involving the iliac wing; type 2, the periacetabular region; type 3 , the pubic rami; and type the sacrum [10]. The internal hemipelvectomies are always required if there is any involvement of the acetabulum. This procedure is a challenging method in orthopedic oncology because of the difficult pelvic anatomy, the concurrence of major neurovascular structures, the reality that pelvic tumors are usually large by the time of diagnosis, and challenges associated with reconstruction. The reconstructive procedure is challenging with a published number of mechanical, infectious, and neurovascular complications of $33 \%$ to $56 \%$ after Type 2 internal hemipelvectomy of a periacetabular tumor [11].

\section{CASE PRESENTATION}

A 48-year-old female has referred to our hospital with a large mass on her groin 2 years ago before admission. Two years ago, the mass was in a peanut size and then grew until it enclosed the genital area. Since then, the patient also began to feel pain that worsens at night. She also complained of walking or standing difficulties due to pain. The patient decided to go to the local public hospital, and then she was transferred to our hospital.

In the physical examination, a hard and painful lump was found at the inguinal region, with a size of $20 \times 15 \times 10$ $\mathrm{cm}$, with a shiny skin around the lump. The lump was hard in consistency, well defined, had an irregular border, warmer than the surrounding, and isolated. There was no tenderness on the lump or any irregularities on the neurovascular function distal to the mass. The movement of hip joint was restricted (Figure 1).

From the radiological examination, in a plain radiograph of pelvic, there was a mass with irregular matrix chondroid from superior and inferior pubic rami with ill-defined border at one-third of the left acetabulum. (Figure 2A). From pelvic MRI, there was mass at left pubic rami and obliterate to the left quadratus muscle, left obturator external muscle, left adductor muscle, iliopsoas muscle and protrude to bowel and vesical urinary, uterus, and rectum to right superolateral. There was also an ill-defined border at one-third left media acetabulum (Figure 2B). Based on this finding, we suspected the mass as a malignant tumor (Figure 2C).

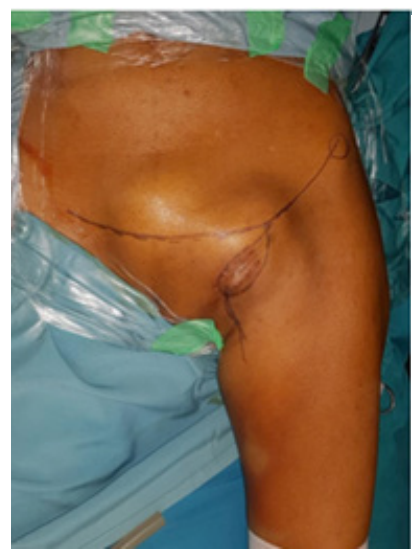

Figure 1. Clinical presentation of the mass in the inguinal region of a 48 -year-old female patient
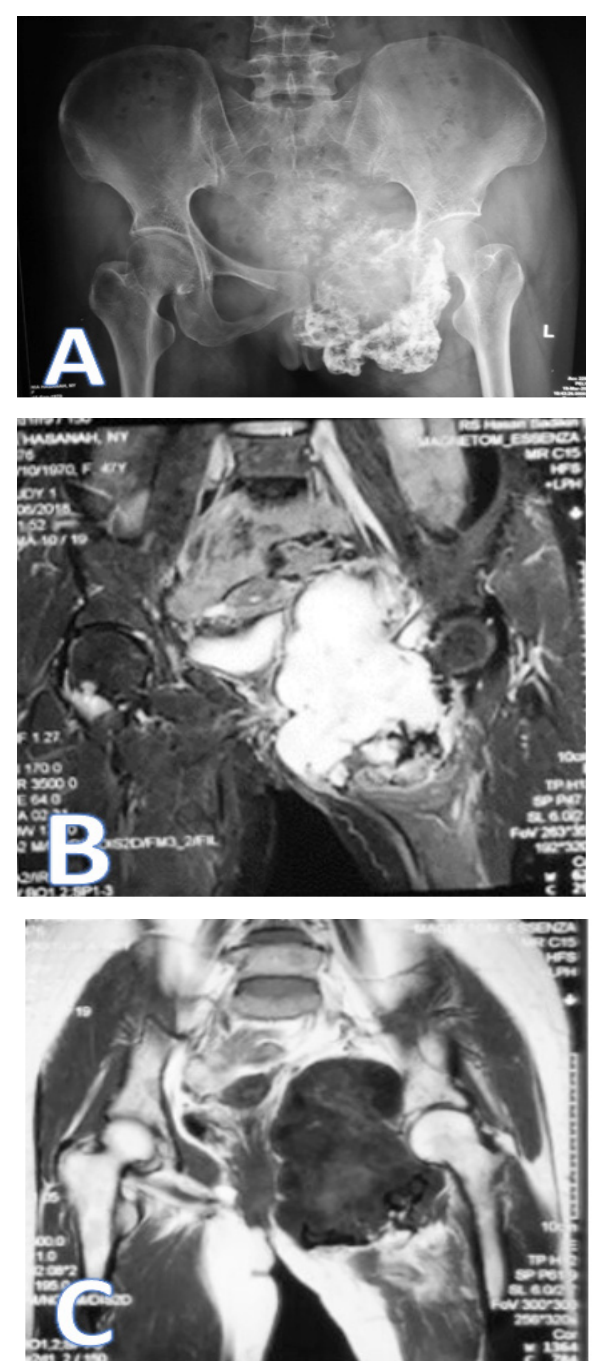

Figure 2. (A) Pelvic AP X-Ray, (B) MRI axial view showed tumor was protruding to retroperitoneal space, (C) MRI coronal view showed tumor was attached to the medial wall of the left acetabulum 
We performed histopathology examination with core biopsy and the conclusion was Chondrosarcoma grade I. In accordance with this conclusion, we, therefore, planned to perform wide resection using type 3 pelvic floor resection followed by bone graft and to maintain pelvic ring we performed an internal fixation with the plate and screw.

On the operation table, the patient was positioned on the supine position. We used the ilioinguinal approach starting from 3-4 cm from the medial margin of the tumor proceeding laterally to ASIS. Afterward, we searched for femoral neurovascular bundles to be preserved. The blunt dissection was then carefully performed to expose the tumor mass and found tumor sized $20 \times 15 \times 10 \mathrm{~cm}$ (Figure 3A). After the tumor mass had been fully identified, we found the tumor arising from superior and inferior pubic rami. The tumor was also attached to the medial wall of the left acetabulum and extended to retroperitoneal area. Superior and inferior pubic rami, medial acetabular wall was osteotomy until healthy tissues because this procedure had a defect at medial acetabulum sized $1 \times 1 \times 0.5 \mathrm{~cm}$ and also head femur exposed (Figure 3B). Afterward, we reconstructed the defect in the medial acetabular with the autograft from iliac and fixation using screwing (Figure $3 \mathbf{C}$ ). To maintain the pelvic ring, we did a fixation using 16 -hole reconstruction plate (Figure 3 D, 4). Then we closed the wound and the drain was inserted. The tumor mass was sent to the Pathology Anatomy for histopathology examination to confirm the diagnosis.
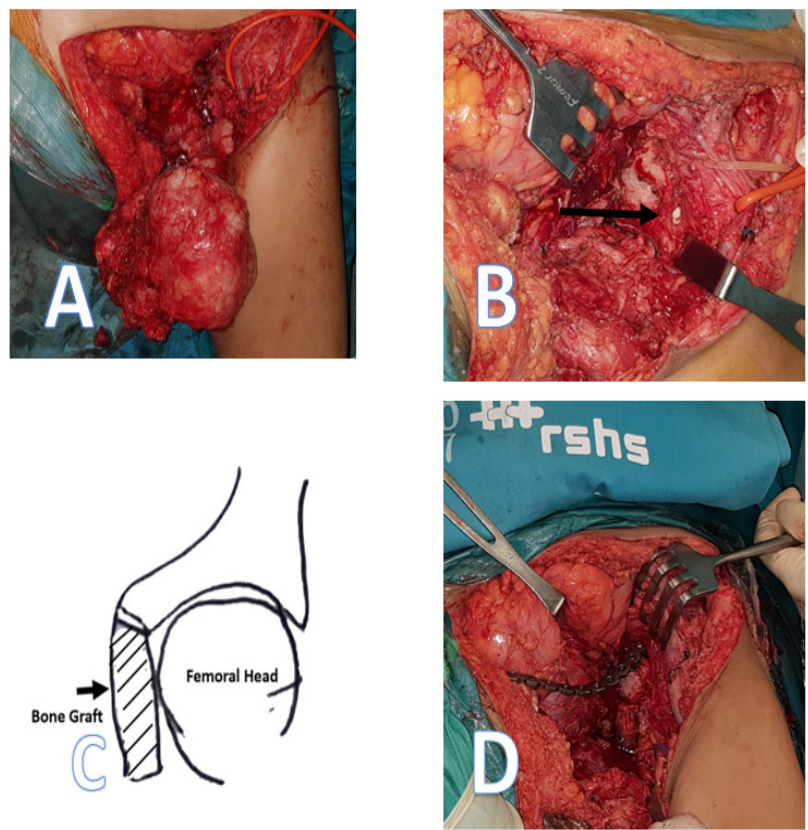

Figure 3. (A) Femoral neurovascular bundle preserved, (B) Medial acetabular wall osteotomy, (C) Covered defect at medial wall using autograft for iliac and fixed using screw, (D) Maintain pelvic ring using LCP - reconstruction plate
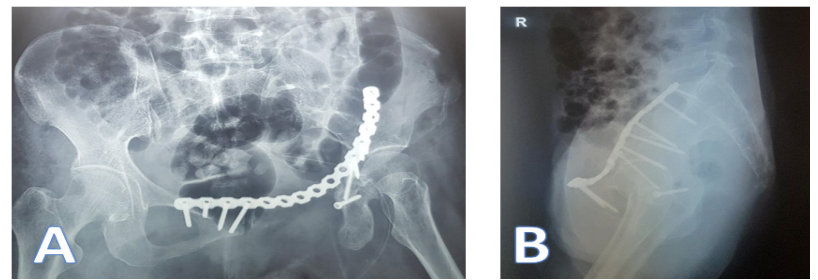

Figure 4. X-Ray post-operation

We did an evaluation of the functional outcome using the Musculoskeletal Tumor Society Score (MSTS) before and after the surgical resection of the tumor. Before the surgical treatment, the MSTS score was 26. Six months after the surgical treatment, the score increased to 30 painless with full weight-bearing, normal gait, no pain, and no restrictions in functional activity as shown in Figure 5.
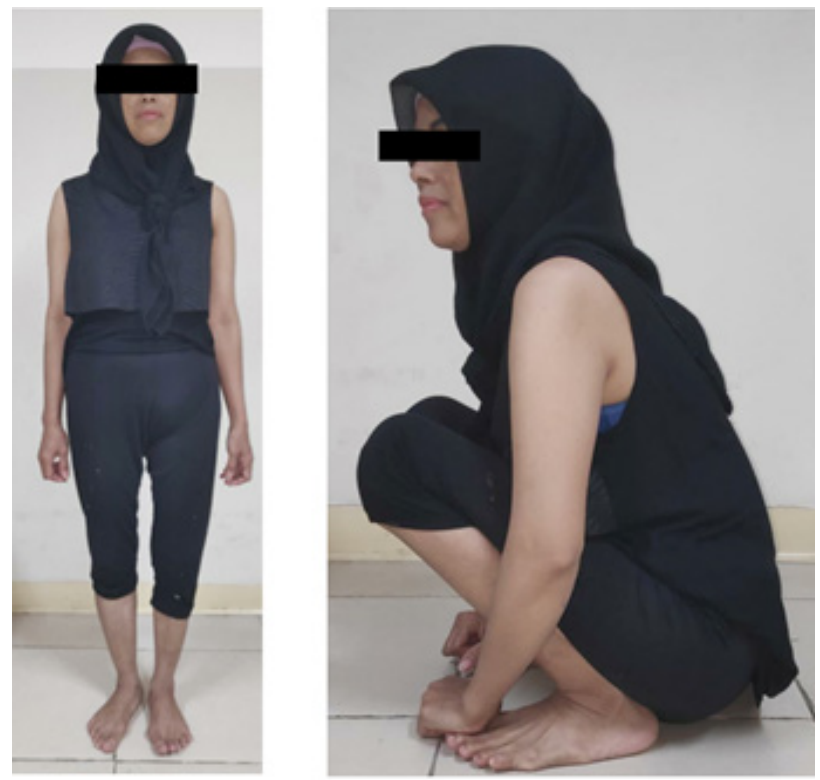

Figure 5. The patient can full weight-bearing and squat

\section{DISCUSSION}

Pelvic chondrosarcoma is the second most common primary malignant bone tumor. The pelvis is the most predominant site in the chondrosarcoma, in between $40-50 \%$ of cases, with the ilium being involved most frequently, followed by the pubis and ischium [2]. However, the rate of limb salvage procedures has risen due to improved preoperative imaging and surgical technique.

Chondrosarcoma of the pelvis remains asymptomatic and may thus be large at the time of diagnosis. Chondrosarcoma is predominantly suffered by mature adults with peak frequency in the fourth decade of life [11]. The age of the patient was concurrent with previous findings regarding incidence. Because there are no anatomical barriers in the pelvis against expansion 
of the tumor, most pelvic sarcomas produce a large extraskeletal mass and must be classified as extracompartmental. An adequate surgical margin (i.e. wide or radical margin) is considered the most important factor associated with local tumor control. In the pelvis, a wide margin is often difficult to obtain due to adjacent visceral, vascular or neural structures. The type of surgical treatment (limb-sparing procedure versus hemipelvectomy) had no influence on the achieved surgical margin. It is the adequacy of the surgical margin and not the type of surgical procedure that is critical for local tumor control [6-8].

Chemotherapy and radiation are not reliably effective in the neoadjuvant or adjuvant setting for classic chondrosarcoma. Therefore, the adequacy of surgical resection determines the patient outcome [6]. Aiming at the complete removal of the tumor, chondrosarcoma of the pelvis is undisputedly one of the most challenging tumors for the orthopedic surgeon. The complex anatomy of the pelvis, with the proximity of neurovascular structures and viscera, adequate resection margins are hardly achieved [1,2,11]. Complications of the surgery and related reconstruction occurred in 51\% of the patients in this series [3].

Management of pelvic tumors that spread toward the acetabulum is multi-aspect. Tumor resection usually attends to a pelvic discontinuity and instability. The extensive range of methods related details to the complexity in handling these cases and receiving excellent surgical results. We consider using type 3 pelvic resection because the main source of tumor from the left and right and there is involvement of one-third of the left medial acetabulum. Type 3 pelvic resection is almost easy and the restoration is usually not needed because acetabulum and axis of weight-bearing are preserved, but there is some defect at the medial acetabulum. which can cause central dislocation from the head femur, so we filled defects using autograft to prevent central dislocation. The graft placed above defect of acetabulum to prevent central dislocation of head femur was fixed using screw. The rotational stability of the pelvic must be thought out because pubic rami are already resected so we manage the rotational stability using reconstruction plate. Histologic studies confirmed the biopsy diagnosis. The margins of the specimen were normal. The microscopic features of the tumor showed intracytoplasmic hyaline globules support chondrosarcoma grade I.

The functional result of the patient was obtained before the surgical treatment and 6 months after the surgical resection of the tumor. An excellent result was achieved in 6 months after the surgical treatment. The MSTS score was 30 showing an excellent result, with good walking ability, no use of supports, normal gait, no pain, no restrictions in functional activity, and satisfactory emotional acceptance.

\section{CONCLUSIONS}

Reconstruction of the pelvis after tumor resection requires a careful preoperative patient evaluation and extensive bone and soft tissue resection to achieve negative tumor margins and stable reconstruction of the osseous and soft tissue defects. Despite the progress in limb salvage in patients with musculoskeletal tumors, pelvic reconstruction remains complicated and the function is always compromised.

\section{DECLARATIONS}

\section{Competing of Interest}

The author declares this research did not receipt any research funding.

\section{Acknowledgment}

There remain no additional people, presents or reserves that need to be supported in this study

\section{REFERENCES}

1. Weber KL, Pring ME, Sim FH. Treatment and outcome of recurrent pelvic chondrosarcoma. Clin Orthop Relat Res. 2002;(397): 19-28.

2. Wirbel RJ, Schulte $M$, Maier B, Koschnik M, Mutschler WE. Chondrosarcoma of the pelvis: oncologic and functional outcome. Sarcoma. 2000;4(4): 161-8.

3. Gökkuş K, Akin T, Sagtas E, Saylik M, Aydın AT. Recurrence of Pelvic Chondrosarcoma through Fascial Defect into Abdominal Cavity. Case Rep Oncol Med. 2014;2014: 674369-

4. Laffosse JM Pourcel A, Reina N, Tricoire JL, Bonnevialle $\mathrm{P}$, Chiron $\mathrm{P}$, Puget J. Primary tumor of the periacetabular region: Resection and reconstruction using a segmental ipsilateral femur autograft. OTSR. 2012;98: 309-318.

5. Gerbers G.G, Jutte P. C. Hip-sparing approach using computer navigation in periacetabular chondrosarcoma. Comput Aided Surg. 2013;18(1-2): 27-32.

6. Gelderblom $H$, Hogendoorn PC, Dijkstra SD, van Rijswijk CS, Krol AD, Taminiau AH, et al. The clinical approach towards chondrosarcoma. The oncologist. 2008;13(3): 320-9.

7. Bovee JV, Hogendoorn PC, Wunder JS, Alman BA. Cartilage tumours and bone development: molecular pathology and possible therapeutic targets. Nat Rev Cancer. 2010;10(7): 481-8. 
8. Giuffrida AY, Burgueno JE, Koniaris LG, Gutierrez JC, Duncan R, Scully SP. Chondrosarcoma in the United States (1973 to 2003): an analysis of 2890 cases from the SEER database. J Bone Joint Surg Am. 2009;91(5): 1063-72.

9. Campanacci DA, Scoccianti G, Franchi A, Roselli G, Beltrami G, Ippolito M, Caff G, Frenos F, Capanna R. Surgical treatment of central grade 1 chondrosarcoma of the appendicular skeleton. J Orthop Traumatol. 2013;14(2): 101-7
10. Enneking WF, Dunham WK. Resection and reconstruction forprimary neoplasms involving the innominate bone. J Bone JointSurg Am. 1978;60: 731-746.

11. Deloin X, Dumaine V, Biau D, Karoubi M, Babinet A, Tomeno B, et al. Pelvic chondrosarcomas: Surgical treatment options. Orthop Traumatol Surg Res. 2009;95(6): 393-401. 